\title{
Variables nutricias asociadas con la ansiedad y la autopercepción corporal en niñas y niños mexicanos de acuerdo con la presencia de sobrepeso/obesidad
}

\author{
Ericka lleana Escalante-lzeta, ${ }^{1}$ Karime Haua-Navarro, ' Luz Irene Moreno-Landa, ${ }^{1}$ Ana Bertha Pérez-Lizaur'
}

Artículo original

\section{ABSTRACT}

\section{Introduction}

Childhood obesity has been associated with the intake of energy-dense foods and sedentary lifestyle, and with mental health indicators such as anxiety and perception of body image. In Mexico it has not been studied the relationship between these variables in children.

\section{Objective}

To assess the body self-perception and risk of anxiety according to the presence of children with normal weight for their stature and age (BMI) and children with overweight/obese, controlling for dietary variables and health habits in children of school age.

\section{Method}

259 boys and girls with normal BMI vs. 326 with overweight/obesity from three public schools in the State of Mexico were evaluated. Diet and lifestyle were associated with the presence of anxiety and body-image dissatisfaction. BMI was calculated from direct measures of weight and height. For anxiety assessment the CMAS-R questionnaire was used and test-SC for body image self-perception.

\section{Results}

$43 \%$ of school children showed cardiovascular risk and $7.7 \%$ metabolic syndrome risk. Children with normal weight have greater satisfaction with their body image and weight, compared to children with overweight or obesity (68.3\% vs. $49.1 \%)$.

\section{Discussion and conclusion}

There were no differences in anxiety according to BM1. The self-perception of body image is less favorable in specific areas of the body, in children with overweight or obesity.

Key words: Body image perception, anxiety, child overweight, obesity.

\section{RESUMEN}

\section{Introducción}

La obesidad infantil se ha asociado con la ingesta de alimentos densamente energéticos, con el sedentarismo y con indicadores de salud mental como la ansiedad manifiesta y la percepción de la imagen corporal. En México no se ha reportado la relación entre dichas variables en población infantil.

\section{Objetivo}

Evaluar la auto-percepción corporal y el riesgo de ansiedad, de acuerdo con la presencia de sobrepeso u obesidad, controlando las variables dietéticas y los hábitos de salud en niños en edad escolar.

\section{Método}

Clasificados por su Índice de Masa Corporal (IMC) se compararon 259 niños y niñas sin sobrepeso/obesidad vs. 326 quienes presentaron dicha condición, en tres escuelas públicas del Estado de México. EI IMC se obtuvo por medición directa del peso y la estatura. Se exploró la relación del consumo de alimentos (dieta) y el estilo de vida con la presencia de ansiedad (cuestionario CMAS-R), auto-percepción y satisfacción corporal (test-SC).

\section{Resultados}

El $43 \%$ de los escolares presentan riesgo cardiovascular y $7.7 \%$ riesgo de síndrome metabólico. Los participantes sin sobrepeso/obesidad presentaron mayor satisfacción con su imagen corporal (68.3\%). No se encontraron diferencias entre el IMC y la ansiedad total, ni con cada una de sus dimensiones.

\section{Discusión y conclusión}

No se reportaron diferencias en la ansiedad de acuerdo con el IMC. La auto-percepción de la imagen corporal fue menos favorable, en zonas específicas del cuerpo en niñas y niños con sobrepeso u obesidad.

Palabras clave: Autopercepción corporal, ansiedad, sobrepeso, obesidad infantil.

Departamento de Salud, Universidad Iberoamericana, Ciudad de México.

Correspondencia: Ana Bertha Pérez Lizaur. Universidad Iberoamericana. Prolongación Paseo de la Reforma. Lomas de Santa Fe, 01219 Ciudad de México. E-mail: anabertha.perez@ibero.mx

Recibido primera versión: 22 de marzo de 2016. Segunda versión: 4 de mayo de 2016. Aceptado: 6 de mayo de 2016. 


\section{INTRODUCCIÓN}

La obesidad infantil es un importante problema de salud pública en el mundo. La Organización Mundial de la Salud (OMS) reporta que para el 2010 afectaba a más de 43 millones de niños en edad prescolar, y para el 2015 se estimaba que 2.3 billones de personas mayores de 15 años presentarían sobrepeso y 700 millones más, obesidad. ${ }^{1}$ En México, la Encuesta Nacional de Salud y Nutrición (ENSANUT) 2012 muestra que el $34.4 \%$ de los niños y niñas, de entre cinco y 11 años de edad, presentan sobrepeso (19.8\%) u obesidad $(14.6 \%) .{ }^{2}$ El estudio de los factores asociados con la presencia de sobrepeso en la población infantil, mismos que son empleados para el tratamiento, se han enfocado principalmente en el desbalance energético (mayor consumo de alimentos vs. menor actividad física), en factores genéticos y perinatales, de estilo de vida sedentario y en factores asociados con el ambiente, caracterizado por la oferta accesible de alimentos densamente energéticos. ${ }^{3}$ Además, se ha documentado la importancia de variables socioculturales, entre las que destacan los estilos de crianza parentales, la influencia de los medios masivos de comunicación y los hábitos familiares de alimentación y de actividad física. ${ }^{4}$ Así mismo, se ha documentado la relación de la obesidad infantil con diversas dimensiones de la salud mental, destacándose la ansiedad y la insatisfacción con la imagen corporal. .-9 $^{5}$

Los trastornos por ansiedad son una de las condiciones psiquiátricas más comunes, presentándose entre un $9 \%$ durante la infancia y en un $32 \%$ en la adolescencia. ${ }^{10}$ Usualmente la ansiedad va de la mano con la depresión $y$ con otros trastornos conductuales ${ }^{11} \mathrm{y}$ se caracteriza por una preocupación excesiva que dura por más de seis meses continuos. ${ }^{12}$ Los síntomas somáticos presentados comúnmente son: cefalea, dolores abdominales, náusea, vómito o diarrea, sensación de ahogo, disnea, taquicardia, opresión torácica, palpitaciones, palidez o rubor, sequedad de la boca, sudoración, inquietud o impaciencia, fatigabilidad, dificultad para concentrarse, irritabilidad, tensión muscular y alteraciones del sueño. ${ }^{13}$ Se ha documentado que la ansiedad en la infancia y adolescencia se caracteriza por un aumento en el apetito y en la ingesta de alimentos, ${ }^{14}$ lo que puede aumentar la incidencia de sobrepeso $\mathrm{u}$ obesidad $\mathrm{y}$, con ello, consecuencias metabólicas como elevación de la tensión arterial e incremento en los niveles de cortisol periférico, entre otros..$^{15,16}$

Por otro lado, se ha encontrado relación entre la insatisfacción con la propia imagen corporal y el aumento del IMC en población infantil femenina, mientras que dicha insatisfacción se reporta en varones cuyos valores de IMC se encuentran en los extremos mínimos o máximos. ${ }^{17}$ Esto ha sido destacado en el proyecto "ETA", un importante estudio que muestra una relación lineal inversa entre los valores continuos de IMC con la satisfacción de la imagen corporal en infantes norteamericanos. ${ }^{18}$
La relación entre la presencia de ansiedad, la insatisfacción con la imagen corporal y el sobrepeso u obesidad pueden, a su vez, asociarse con la ingesta de alimentos que aportan mayor energía. Esto puede deberse a una alta sensibilidad para la obtención de recompensas a corto plazo y a la presencia de una auto-regulación disfuncional. ${ }^{15}$ Así mismo, se ha documentado que la disminución funcional de la dopamina, puede llevar al individuo a la compensación de la gratificación inmediata, a través del consumo de alimentos densamente energéticos, ricos en hidratos de carbono. ${ }^{19}$

Considerando las prevalencia alarmantes de obesidad infantil en México, resulta importante analizar la relación entre la presencia de sobrepeso/obesidad con la ansiedad manifiesta, la insatisfacción con la imagen corporal y el consumo de alimentos, con la finalidad de hacer sugerencias concretas para su atención. La hipótesis se centra en el supuesto de que las niñas y niños con IMC clasificado con sobrepeso/obesidad, presentarán mayor riesgo de desarrollar ansiedad y menor satisfacción con su imagen corporal.

El objetivo del presente estudio es evaluar la auto-percepción corporal y el riesgo de ansiedad de acuerdo con la presencia de sobrepeso $u$ obesidad, controlando las variables dietéticas y los hábitos de salud durante la edad escolar.

\section{MÉTODO}

\section{Diseño}

Estudio transversal, comparativo. El Departamento de Salud de la Universidad Iberoamericana desarrolló, implementó y evaluó durante el año 2013 una intervención diseñada para la rehabilitación de hábitos en alimentación, actividad física y descanso dirigida a niñas y niños en edad escolar. ${ }^{20} \mathrm{El}$ presente trabajo constituye una parte de la evaluación basal del estudio en mención.

\section{Muestra}

Se trabajó con 585 estudiantes de tres escuelas primaria públicas urbanas de Toluca, Estado de México. El rango de edad de los escolares participantes fue de ocho a 12 años, considerando que de acuerdo con los datos nacionales, el aumento de prevalencias de sobrepeso/obesidad sucede durante los últimos años de la escuela primaria. ${ }^{2}$

Las escuelas se seleccionaron a partir de los resultados del V Censo de Peso y Talla en el Estado de México que se realizó en el ciclo escolar 2009-2010. ${ }^{21}$ De 10 escuelas primarias seleccionadas con mayor prevalencia de obesidad, aceptaron participar tres, de las cuales se reportan los datos de la evaluación basal en el presente estudio. 


\section{Procedimiento}

Sólo se incluyeron a los escolares del tercer al sexto grado, quienes desearan participar en el estudio y que contaran con la autorización de sus padres y/o madres, con la firma de la carta de ética, confidencialidad y consentimiento informado.

Nutriólogas previamente capacitadas y estandarizadas se encargaron de tomar las mediciones antropométricas de peso, estatura y circunferencia de cintura, así como de aplicar los cuestionarios de auto-reporte dentro del contexto y horario escolar, de manera individual, en una sección autorizada por la directora y bajo la supervisión de una profesora.

Para la aplicación de los cuestionarios se formaron grupos de 10 escolares y un facilitador leyó en voz alta cada reactivo, dando tiempo suficiente para responder de forma individual.

\section{Instrumentos}

A partir de los datos se calculó el IMC, considerando el peso, la talla y la edad, tomando como referente las tablas de la OMS.22 Igualmente se calculó el Índice Cintura Estatura (ICE) ${ }^{23}$ y el perímetro de cintura como indicadores de riesgo cardiovascular y de síndrome metabólico durante la infancia, de acuerdo con los criterios de la Federación Internacional de Diabetes (IDF). ${ }^{24}$ Para la evaluación dietética y hábitos de salud se elaboró un cuestionario auto-aplicado que indagó la frecuencia y hábitos de consumo de alimentos, hábitos relacionados con la salud como tiempo de actividad física, horas frente a pantallas (tv. y videojuegos) y horas de sueño.

El cuestionario integrado por 37 reactivos reportaba cada ítem bajo cinco opciones de respuesta: Nunca, un día a la semana, dos a cuatro días a la semana y cinco a siete días a la semana. La primera sección registraba el consumo de refrescos, jugos de fruta, agua de fruta con azúcar, bebidas preparadas con polvos azucarados y saborizantes, galletas, pastelillos, pan dulce, botanas o frituras (chicharrones/papas), dulces (caramelos/paletas), chocolate, polvos dulces para la leche de sabores (chocolate, fresa o vainilla), verduras, frutas, guisados fritos, agua simple, leche entera, leche entera azucarada y con sabores (chocolate, fresa o vainilla).

La segunda parte del cuestionario incluyó ítems que exploraban conductas alimentarias y de salud. Se cuestionó la frecuencia con que el niño percibe que come de más, el tamaño de las porciones (grandes, pequeñas o suficientes), ejecución de juegos activos con amigos o con su familia, práctica de algún deporte, tiempo de ver televisión en horas de comida, horas de sueño, desayunar antes de ir a la escuela y la compra de alimentos dentro de la escuela o en sus alrededores.
Para la evaluación de ansiedad se usó la Escala de Ansiedad Manifiesta (CMAS-R) de Reynolds y Richmond, ${ }^{25}$ probada previamente en población mexicana. ${ }^{26} \mathrm{El}$ instrumento cuenta con 37 reactivos de respuesta dicotómica (si) no), diseñada para valorar el nivel y la naturaleza de la ansiedad en niños y adolescentes. La suma de las respuestas positivas determina la puntuación de ansiedad total, misma que se traduce a una escala por percentiles de acuerdo con la edad y el sexo de los participantes. A su vez, proporciona cuatro calificaciones en dimensiones adicionales: ansiedad fisiológica, inquietud o hipersensibilidad, preocupaciones sociales o concentración y mentira.

El instrumento para medir el grado de percepción/satisfacción corporal fue el test-SC de García-Tornel y Gaspar, adaptado por Raich, Torras y Sánchez en el año $2001 .^{27} \mathrm{El}$ test consiste en el dibujo de una persona con 18 partes corporales, donde el niño autoevalúa su agrado por su propio cuerpo (auto-aprobación total). Usa una escala entre 0 a 10; donde 0 significa estar completamente a disgusto con esa parte del cuerpo y 10 totalmente conforme. Los 18 ítems se promedian para obtener un índice de satisfacción corporal general. ${ }^{27}$

\section{Análisis estadístico}

Se describieron variables demográficas (medidas de tendencia central, frecuencias y proporciones) y posteriormente se realizaron comparaciones bi-variadas entre el IMC con las variables independientes planteadas (ansiedad e insatisfacción con la imagen corporal), además de evaluarla con sus respectivas co-variables (consumo de alimentos y hábitos de salud) ya que ambas han sido ampliamente documentadas en el área de la nutrición como factores de riesgo para presentar sobrepeso $u$ obesidad. ${ }^{4,7,28}$ Diversos estudios epidemiológicos recurren a la clasificación de sobrepeso/obesidad infantil en una sola categoría para facilitar la comparación entre grupos, considerando que el riesgo metabólico por exceso de adiposidad se inicia con la clasificación de sobrepeso. ${ }^{4}$

Así mismo, se consideraron comparaciones antropométricas que son referencia de comorbilidades asociadas al sobrepeso/obesidad, como el riesgo cardiovascular y el riesgo metabólico durante la infancia (indice de cintura cadera circunferencia de cintura). La comparación de variables continuas se hizo por medio de la $t$-student para muestras independientes y para la comparación de proporciones se utilizó la prueba $\chi^{2}$.

Para complementar el análisis se propusieron modelos de regresión logística para explicar la presencia de alteraciones en cada una de las dimensiones de ansiedad (como variables dependientes), considerando las co-variables dietéticas, de estilo de vida, sexo y edad. Como se verá en la sección de resultados no se consideró al IMC en su clasificación dicotómica (con o sin sobrepeso/obesidad) como parte 
Cuadro 1. Características descriptivas de la muestra

\begin{tabular}{lc}
\hline Variable & $\%(n)$ \\
\hline Sexo & \\
Masculino & $50.8(297)$ \\
Femenino & $49.2(288)$ \\
Diagnóstico de estado de nutrición por IMC & \\
Bajo peso & $2.2(13)$ \\
Peso normal & $42.1(246)$ \\
Sobrepeso & $29.7(174)$ \\
Obesidad & $26.0(152)$ \\
Presencia de adiposidad central (CC)* & $7.7(45)$ \\
Presencia de riesgo cardiovascular (ICE) * & $25.0(146)$ \\
Presencia de ansiedad manifiesta & $20.9(122)$ \\
Presencia de ansiedad fisiológica & $34.7(203)$ \\
Presencia de inquietud & $24.6(144)$ \\
Presencia de preocupación & $32.6(191)$ \\
Presencia de mentira & $42.4(248)$ \\
\hline
\end{tabular}

* Circunferencia de Cintura.

* * Índice Cintura Estatura.

de dichos modelos ya que en los análisis bi-variados no se encontraron diferencias estadísticamente significativas entre los grupos.

\section{RESULTADOS}

Se evaluaron 585 niños y niñas de primaria, no reportándose pérdidas en esta primera recolección de datos. El 50.8\% de los participantes eran varones y el promedio de edad fue de $9 \pm 1.32$ años. Un poco más de la mitad de ellos (57.7\%) presentaban sobrepeso $u$ obesidad. Las características generales de las variables antropométricas y psicológicas evaluadas se muestran en el cuadro 1.

Se identificó con riesgo para síndrome metabólico, por presentar una cintura demasiado prominente, al $7.7 \%$ de los participantes, todos ellos con sobrepeso/obesidad. Algunos autores han propuesto al ICE como un buen indicador de riesgo cardiovascular infantil cuando sus valores se encuentran por arriba de $0.5 .{ }^{22}$ Considerando este criterio, el $43 \%$ de los participantes fueron clasificados con riesgo; sólo un niño con peso normal fue incluido en este grupo.

Sobre la satisfacción de la imagen corporal, se identificaron diferencias en los puntajes promedio sobre cuánto refirieron los participantes que les gustaban diferentes partes de su cuerpo. Como puede observarse en el cuadro 2, el grupo sin sobrepeso/obesidad refiere que le agradan ciertas partes de su cuerpo de una forma significativamente mayor que el grupo con sobrepeso/obesidad, como sus abdómenes (9.0 vs. 8.42), sus brazos (9.24 vs. 8.92), su cintura (9.12 vs. 8.6) y sus muslos (9.20 vs. 8.86). De igual modo, se identificaron diferencias significativas en la frecuencia con la que el grupo de peso normal $(68.3 \%)$ vs. el grupo con sobrepeso/obesidad (49.1\%) refiere estar conforme con su peso.
Cuadro 2. Variables de satisfacción corporal de acuerdo con la presencia de sobrepeso/obesidad clasificado por IMC

\begin{tabular}{|c|c|c|c|}
\hline & \multicolumn{2}{|c|}{ Sobrepeso/Obesidad } & \multirow[b]{2}{*}{ Sig. * } \\
\hline & $\frac{\operatorname{Sin}}{\bar{x}, D E}$ & $\frac{\text { Con }}{\bar{x}, \mathrm{DE}}$ & \\
\hline Me gusta mi cabello & $9.58(1.25)$ & 9.52 (1.49) & 0.63 \\
\hline Me gustan mis ojos & $9.18(1.86)$ & $9.17(1.93)$ & 0.95 \\
\hline Me gusta mi cutis & $8.33(2.53)$ & $8.52(2.45)$ & 0.34 \\
\hline Me gusta mi nariz & $8.94(4.83)$ & $8.81(2.24)$ & 0.70 \\
\hline Me gusta mi boca & $9.22(1.65)$ & $9.13(1.89)$ & 0.54 \\
\hline Me gustan mis labios & $9.04(2.01)$ & $8.98(2.11)$ & 0.72 \\
\hline Me gusta mi cuello & 9.09 (1.73) & 9.00 (2.09) & 0.55 \\
\hline Me gusta mi pecho & $9.03(2.00)$ & $8.89(2.24)$ & 0.41 \\
\hline Me gustan mis brazos & $9.24(1.76)$ & $8.92(2.16)$ & 0.05 \\
\hline Me gustan mis manos & $9.13(1.88)$ & $9.03(2.08)$ & 0.52 \\
\hline Me gusta mi abdomen & $9.00(2.09)$ & $8.42(2.81)$ & 0.04 \\
\hline Me gusta mi cintura & $9.12(1.98)$ & $8.60(2.48)$ & 0.00 \\
\hline Me gustan mis genitales & $8.93(3.06)$ & $8.60(2.75)$ & 0.16 \\
\hline Me gustan mis nalgas & $8.45(2.64)$ & $8.29(3.00)$ & 0.50 \\
\hline Me gustan mis caderas & $9.03(2.05)$ & $8.86(2.37)$ & 0.35 \\
\hline Me gustan mis muslos & $9.20(1.90)$ & $8.86(2.27)$ & 0.05 \\
\hline Me gustan mis piernas & $9.01(2.00)$ & $9.40(5.45)$ & 0.23 \\
\hline Me gusta mi piel & $9.21(1.92)$ & $9.08(2.13)$ & 0.45 \\
\hline
\end{tabular}

* T-Student muestras independientes.

Los datos de ansiedad reportaron una prevalencia del $20.9 \%$. Se presentó ansiedad fisiológica en un $34.7 \%$; inquietud, $24.6 \%$; preocupación, $32.6 \%$ y mentira en un $42.4 \%$. La prevalencia no fue distinta al segmentarla por presencia de sobrepeso/obesidad para ninguno de los rasgos evaluados (cuadro 3), por lo que no se planteó un análisis predictivo considerando las variables anteriores.

Los hallazgos fueron distintos al correlacionar la ubicación por percentiles de los puntajes de ansiedad, inquietud, preocupación y mentira con la medición del perímetro de cintura, identificándose algunas correlaciones significativas, aunque muy débiles, para algunas variables. Se encontró que a mayor medición de cintura mayores serán los puntajes en las dimensiones ${ }^{25}$ de ansiedad fisiológica $(r=0.111 ; p=0.008)$, de inquietud $(r=0.110 ; p=0.015)$, de preocupación $(r=0.100 ; p=0.015)$ y de mentira $(r=0.144$; $p=0.000)$.

Cuadro 3. Variables de ansiedad, inquietud, preocupación y mentira comparando población con y sin sobrepeso/obesidad

\begin{tabular}{|c|c|c|c|c|}
\hline & \multicolumn{2}{|c|}{ Sobrepeso/Obesidad } & \multirow[b]{2}{*}{$\begin{array}{c}\mathrm{T} \\
\mathrm{GL} \\
\end{array}$} & \multirow[b]{2}{*}{ Sig. * } \\
\hline & $\begin{array}{c}\operatorname{Sin} \\
(n=259) \\
\%\end{array}$ & $\begin{array}{c}\text { Con } \\
(n=326) \\
\%\end{array}$ & & \\
\hline Ansiedad total & 23.2 & 19.0 & 1.5043 & 0.220 \\
\hline Ansiedad fisiológica & 37.8 & 32.2 & 2.0184 & 0.155 \\
\hline Inquietud & 25.2 & 24.2 & 0.0580 & 0.810 \\
\hline Preocupación & 32.0 & 33.1 & 0.0769 & 0.782 \\
\hline Mentira & 40.9 & 43.6 & 0.4093 & 0.522 \\
\hline
\end{tabular}

* Prueba: $\chi^{2}$ 
Cuadro 4. Variables predictoras de ansiedad y sus dimensiones

\begin{tabular}{|c|c|c|c|}
\hline Dimensión & Factor de riesgo* & $O R * *$ & $\begin{array}{c}\text { Intervalo de } \\
\text { Confianza } 95 \%\end{array}$ \\
\hline \multicolumn{4}{|c|}{$\overline{\text { Ansiedad total }}$} \\
\hline & Consumo de pan dulce & 1.29 & $1.01-1.65$ \\
\hline & Sentí que comí de más & 1.46 & $1.14-1.86$ \\
\hline & Consumo de polvos dulces & 0.08 & $0.64-0.99$ \\
\hline \multicolumn{4}{|c|}{ Ansiedad fisiológica } \\
\hline & Sentí que comí de más & 1.54 & $1.22-1.94$ \\
\hline & Sentí que comí porciones pequeñas & 1.33 & $1.10-1.61$ \\
\hline & Comprar alimentos en la escuela & 1.32 & $1.07-1.61$ \\
\hline & Consumo de polvos dulces & 0.72 & $0.54-0.88$ \\
\hline \multicolumn{4}{|l|}{ Inquietud } \\
\hline & Sentí que comí de más & 1.55 & $1.21-1.98$ \\
\hline & Sentí que comí porciones pequeñas & 1.46 & $1.18-1.81$ \\
\hline \multicolumn{4}{|c|}{ Preocupación } \\
\hline & Sentí que comí de más & 1.44 & $1.15-1.81$ \\
\hline & Sentí que comí porciones pequeñas & 1.36 & $1.12-1.64$ \\
\hline & Consumo de polvos dulces & 0.78 & $0.64-0.95$ \\
\hline & Jugar en el recreo & 0.76 & $0.62-0.92$ \\
\hline & Dormir de 8 a 10 horas & 0.81 & $0.68-0.96$ \\
\hline \multicolumn{4}{|l|}{ Mentira } \\
\hline & Consumo de jugos de frutas & 1.57 & $1.25-1.97$ \\
\hline & Comprar alimentos en la escuela & 1.30 & $1.05-1.61$ \\
\hline & Índice cintura/estatura & 2.75 & $1.28-5.92$ \\
\hline
\end{tabular}

Al usarse el IMC como variable continua también se asoció significativa y positivamente sólo con los puntajes de la dimensión "mentira" ( $r=112 ; p=0.007)$.

Se exploraron algunas variables dietéticas y del estilo de vida en un modelo de predicción para la presencia de ansiedad y sus dimensiones. El cuadro 4 muestra los factores predictivos, encontrados como estadísticamente significativos, para cada una de las dimensiones de ansiedad. ${ }^{25}$ Para ansiedad total se identificaron como factores de riesgo: la edad $(\mathrm{OR}=1.30)$, la frecuencia de consumo de pan dulce $(\mathrm{OR}=1.29)$ y la frecuencia con que el niño siente que comió de más $(\mathrm{OR}=1.46)$. Se identificó como comportamiento protector la frecuencia de consumo de polvos dulces para leche $(\mathrm{OR}=0.08)$.

Para las demás dimensiones coinciden como factores de riesgo, principalmente, la sensación de comer de más, la sensación de comer porciones pequeñas, comprar alimentos en el colegio y el consumo de polvos dulces para leche (cuadro 4).

\section{DISCUSIÓN Y CONCLUSIÓN}

Considerando los resultados obtenidos en el presente estudio, es posible concluir que la clasificación de sobrepeso/obesidad a través del IMC infantil, no permite reportar diferencias en los indicadores de ansiedad. Sobre la autopercepción corporal se registraron diferencias entre grupos, en las zonas corporales que suelen tener más reservas adiposas en caso de presentar sobrepeso/obesidad. Por otro lado, se encontró correlación positiva entre el perímetro de la cintura con los puntajes de ansiedad fisiológica, preocupación y mentira, lo que puede traducirse como un mejor indicador antropométrico para hacer comparaciones con las dimensiones de ansiedad infantil. Corroborando estos resultados, Vámosi, ${ }^{28}$ en una extensa revisión de la literatura científica, menciona que la obesidad infantil se relaciona con diversos factores psicosociales entre los que destaca la ansiedad, misma que se sugiere debe ser abordada dentro de tratamientos integrales.

En el grupo de niñas y niños con peso normal se identificó mayor aceptación y agrado de su cuerpo en comparación con el grupo que presentaba sobrepeso/obesidad. Esto mismo fue reportado por otros estudios, donde la obesidad infantil se asocia con la insatisfacción corporal, principalmente el disgusto hacia la propia figura, lo que puede desencadenar depresión. ${ }^{17,29}$ Esto puede explicarse, en parte, por las creencias y juicios de valor demeritados, otorgados al niño con obesidad bajo un modelo occidental de representación del cuerpo. ${ }^{30}$ Es decir, el niño con sobrepeso puede ser objeto de críticas y burlas originadas por su composición corporal, lo que a mediano plazo puede llevarle a rechazar su propia imagen, presentar conductas alimentarias de riesgo, con un posible desenlace en trastornos de la conducta alimentaria. ${ }^{31}$

La presión social y el acoso a los escolares con obesidad puede causar ansiedad o, a la inversa, la ansiedad puede lle- 
varlo a la sobre-ingesta de alimentos, lo que a su vez incide en la ganancia de peso, relacionada con la insatisfacción con la imagen corporal, ${ }^{32}$ por lo que dicho proceso se vuelve un círculo vicioso recurrente.

Como parte de los resultados, se identificaron algunos factores protectores de la presencia de ansiedad, como el consumo de polvos dulces y con saborizantes artificiales para agregar a la leche, jugar en el recreo y dormir 10 horas (dimensión de preocupación). En un estudio realizado por Pervanidou $^{16}$ se documentó la estrecha relación negativa entre las horas de sueño y las concentraciones de cortisol, como un indicador de ansiedad, en infantes escolares con obesidad. Esto podría explicar que niñas y niños con hábitos adecuados de sueño reporten puntajes menores en la dimensión de "preocupación". ${ }^{25}$ Lo cual corrobora que los hábitos adecuados de vida y salud de los infantes y no sólo de alimentación, pueden tener impactos positivos en su estado de nutrición y en su salud mental. Igualmente, la dimensión de "mentira"25 aumenta con la frecuencia de consumos específicos (como jugos de frutas, pan dulce), comportamientos alimentarios (consumir alimentos dentro de la escuela, mismos que suelen ser de alto valor energético), la sensación de comer porciones pequeñas y la sensación del niño de comer de más. Treviño, ${ }^{33}$ encontró también, en población infantil mexicana, una relación positiva entre el consumo de monosacáridos y disacáridos con el cortisol sérico, como un indicador de ansiedad. Esto puede dar pequeños indicios de la necesidad de estudiar la asociación del consumo de alimentos ricos en hidratos de carbono con la presencia de sobrepeso/obesidad y, a su vez, denotar la presencia de ansiedad en población infantil mexicana.

Como es notorio en los resultados, las dimensiones específicas de ansiedad aumentaron con el consumo de alimentos dulces. Diversos estudios realizados por Schulte y Avena, ${ }^{34}$ sugieren que el consumo de alimentos altamente procesados comparten las propiedades de algunas citoquinas farmacéuticas, comparando el abuso de drogas con la rápida absorción de hidratos de carbono refinados, reportados por el índice glicémico de alimentos ricos en grasas e hidratos de carbono. Esto aporta resultados preliminares que sugieren propiedades adictivas en alimentos ricos en azúcares. ${ }^{34}$ Con estos antecedentes y considerando nuestros resultados sobre el consumo de polvos dulces y jugos, podría suponerse que las alteraciones de ansiedad se asocian a la búsqueda de gratificaciones inmediatas por medio del consumo de hidratos de carbono de absorción rápida, que a su vez pueda ser aceptada y permitida por la familia y el grupo social de referencia.

Dichos mecanismos igualmente podrían regular la preferencia de alimentos dulces bajo condiciones altamente estresantes. Esto puede ir de la mano con el concepto de comer emocional, estudiado por Goossens L, ${ }^{14}$ quien reportó una estrecha relación entre la presencia de ansiedad, obesidad y la pérdida de control al comer en adolescentes; relación que se encuentra mediada por los estilos de afrontamiento al estrés y se presenta especialmente en el sexo femenino. En el caso de nuestros resultados, la percepción de consumir porciones pequeñas podría denotar la pérdida de control sobre las cantidades de alimento que realmente se desean comer.

Finalmente, se identificaron limitaciones metodológicas en cuanto al tamaño de la muestra, así como la aplicación grupal de los instrumentos de salud mental. Así mismo, los resultados finales no son extrapolables a contextos diferentes de la región centro de México, donde se comparten gustos y hábitos alimentarios específicos.

Para futuras investigaciones se recomienda complementar los datos reportados por los escolares con la ansiedad manifestada por sus padres. Tanto los hábitos como algunas características de salud mental se han asociado con la respuesta infantil caracterizada por procesos de imitación, propios de su etapa de desarrollo. Así mismo, se recomienda evaluar los estilos de afrontamiento en la infancia, como variable de control a la respuesta de ansiedad. También se recomienda usar como parámetros antropométricos de desenlace al ICE y la medición de la circunferencia de cintura.

\section{Agradecimientos}

Agradecemos a las escuelas que nos permitieron realizar los talleres del programa de intervención y al Sistema de Desarrollo Integral de la Familia (DIFEM), por el apoyo económico a las facilitadoras para llevar a cabo el programa. A María José Calva Corona por su apoyo en la construcción de la base de datos.

\section{Financiamiento}

Este estudio fue financiado por el Departamento de Salud de la Universidad Iberoamericana y el Sistema para el Desarrollo Integral de la Familia del Estado de México.

\section{Conflicto de intereses}

Los autores declaran no tener ningún conflicto de intereses.

\section{REFERENCIAS}

1. Onis M, Blossner M, Borghi E. Global prevalence and trends of overweight and obesity among preschool children. Am J Clin Nutr 2010;92:1257-1264.

2. Gutiérrez JP, Rivera-Dommarco J, Shamah-Levy T, Villalpando-Hernández $S$ et al. Encuesta Nacional de Salud y Nutrición 2012. Resultados nacionales. Cuernavaca, México: Instituto Nacional de Salud Pública; 2012.

3. Ebbeling c, Pawlak D, Ludwig D. Childhood obesity: public-health crisis, common sense cure. Lancet 2002;360:473-482.

4. Mahmood L. The childhood obesity epidemic: A mini review. Int J Med Public Health 2015;5:6-9.

5. Rawana J, Morgan A, Nguyen H, Craig S. The relation between eating and weight-related disturbances and depression in adolescence: a review. Clinical Child Family Psychology Review 2010;13(3):213-230.

6. Gariepy G, Nitka D, Schmitz N. The association between obesity and anxiety disorders in the population: a systematic review and meta-analysis. International J Obesity 2010;34(3):407-419. 
7. Hemmingsson E. A new model of the role of psychological and emotional distress in promoting obesity: conceptual review with implications for treatment and prevention. Obesity Reviews 2014;15:769-779.

8. Claus L, Braet C, Decaluw V. Dieting history in obese youngsters with and without disordered eating. International $\mathrm{J}$ Eating Disorders 2006;39(8):721-728.

9. Decaluw' V, Braet C. Prevalence of binge-eating disorder in obese children and adolescents seeking weight-loss treatment. International J Obesity 2003;27(3):404-409.

10. Essau CA, Gabbidon J. Epidemiology, comorbidity and mental health service utilization. En: Essau CA, Ollendick TH (eds.). The Wiley-Blackwell handbook of the treatment of childhood and adolescent anxiety. Primera edición. Chichester: Wiley-Blackwell, 2013; pp23-42.

11. Kim-Cohen J, Caspi A, Moffitt TE et al. Prior juvenile diagnoses in adults with mental disorder: developmental follow-back of a prospective-longitudinal cohort. Arch Gen Psychiatry 2003;60:709-717.

12. Creswell C, Waite $P$, Cooper $P$. Assessment and management of anxiety disorders in children and adolescents. Arch Dis Child 2014;99:674-678.

13. Mardomingo Sanz MJ, Características clínicas de los trastornos de ansiedad. Revista Pediatría Atención Primaria 2001;3(10):237-247.

14. Goossens L, Braet C, Van Vlierberghe L, Mels S. Loss of control over eating in overweight youngsters: the role of anxiety, depression and emotional eating. Eur Eat Disord Rev 2009;17:68-78.

15. Puder JJ, Munsch S. Psychological correlates of childhood obesity. International J Obesity 2010;34:S37-S43.

16. Pervanidou P, Chrousos G. Stress and obesity/metabolic syndrome in childhood and adolescence. International J Pediatric Obesity 2011;6(S1):21-28.

17. Russell-Mayhew Sh, McVey G, Bardick A, Ireland A. Mental health, wellness and childhood overweight/obesity. J Obesity 2012;junio 24, doi. 10.1155/2012/281801

18. Austin SB, Haines J, Veugelers P. Body satisfaction and body weight: gender differences and sociodemographic determinants. BMC Public Health 2009;9:313.

19. Stice E, Spoor S, Ng J, Zald DH. Relation of obesity to consummatory and anticipatory food reward. Physiol Behav 2009;97:551-560.

20. Pérez- Lizaur AB. Un dos tres por nuestra salud. México. México D.F [citado en abril 2015]. Disponible en: http://123xnuestrasalud.ibero. $\mathrm{mx} /$ programas.php?seccion=salud

21. Ávila A, Chávez A, Quiroz M, Juárez L et al. La desnutrición infantil en el Estado de México, 1994-2010. Resultado del quinto censo de talla y peso. Toluca: Consejo Estatal de Población; 2012.
22. OMS. Patrones de crecimiento infantil de la OMS [Internet]. 2015 [citado 12 april 2015]. Disponible en: http://www.who.int/childgrowth/es/

23. Bener A, Yousafzai M, Darwish S, Al-Hamaq A et al. Obesity Index That Better Predict Metabolic Syndrome: Body Mass Index, waist circumference, waist hip ratio, or waist height ratio. J Obes 2013;agosto 13:1-9, doi. 10.1155/2013269038.

24. International Diabetes Federation. Criteria of Metabolic Syndrome in Children and Adolescents [Internet]. 2011 [citado 12 abril 2015]. Disponible en: http://www.idf.org/metabolic-syndrome/children/criteria

25. Reynolds C, Richmond B. Escala de ansiedad manifiesta en niños: (revisada) CMAS-R. México: Editorial El Manual Moderno; 1997.

26. De la Torre MI, Cubillas-Rodríguez MJ, Román-Pérez R, Valdez EA. Ideación suicida en población escolarizada infantil: factores psicológicos asociados, Salud Ment 2009;32:495-502.

27. Raich R, Mora M, Soler A, Ávila C et al. Adaptación de un instrumento de evaluación de la insatisfacción corporal. Clínica Salud 1996;1(7):51-66.

28. Vámosi M, Heitmann L, Kyvik $O$. The relation between an adverse psychological and social environment in childhood and the development of adult obesity: a systematic literature review. Obesity Reviews 2010;11:177-184.

29. Allen K, Byrne S, Blair E, Davis E. Why do some overweight children experience psychological problems? The role of weight and shape concern. International J Pediatric Obesity 2006;1(4):239-247.

30. Field A, Cartwright S, Reynolds Sh, Creswell C. Future directions for child anxiety theory and treatment. Cognition Emotion 2008;22(3):385-394

31. Neumark-Sztainer D. Preventing the broad spectrum of weightrelated problems: working with parents to help teens achieve a healthy weight and a positive body image. J Nutr Educ Behav 2005;37(suppl 2):S133-S139.

32. Evans R, Roy J, Geiger B, Werner $\mathrm{K}$ et al. Ecological strategies to promote healthy body image among. Children J School Health 2008;78(7):359-367.

33. Treviño Villareal V. López Guevara LE, Ramírez López, Tijerina Sáenz A. Relación de cortisol sérico con los componentes del síndrome metabólico, ingesta alimentaria y trastorno de ansiedad en niños de 8 a 12 años con obesidad. Nutr Hosp 2012;27(5):1562-1568

34. Schulte E, Avena N, Gearhardt A. Which foods may be addictive? The roles of processing, fat content, and glycemic load. Plos ONE 2015;10(2):1-18. 